\title{
Évaluation des activités antifongiques des extraits de graines de Thevetia peruviana contre Phytophthora colocasiae (Oomycètes) agent causal du mildiou du taro (Colocasia esculenta (L.) Schott) au Cameroun
}

\author{
Essomé Salé Charles ${ }^{1}$, Ngoh Dooh Jules Patrice ${ }^{2}$, Heu Alain ${ }^{3}$, Ndogho Pegalepo Angèle1, Ngatsi \\ Zemko Patrice ${ }^{1}$, Chewachong Godwill ${ }^{4}$ and Ambang Zachee ${ }^{1^{*}}$ \\ 1 Laboratory of Phytopathology and Crop Protection, Department of Plant Biology, University of Yaounde I, PO Box \\ 812, Yaounde-Cameroon \\ ${ }^{2}$ Department of Biological Science, Faculty of Science, University of Maroua, Cameroon \\ ${ }^{3}$ Department of Agriculture, High Technical College of Ebolowa, University of Yaounde I, Cameroon \\ ${ }^{4}$ Department of Crop Protection, Faculty of Agriculture, University of Dschang, Cameroon \\ ${ }^{*}$ Corresponding author: Pr Z. AMBANG; E-mail: zachambang@yahoo.fr
}

Original submitted in on $18^{\text {th }}$ May 2020. Published online at www.m.elewa.org/journals/ on $31^{\text {st }}$ July 2020 https://doi.org/10.35759/JABs.151.7

\section{RESUME}

Objectif : Le mildiou du taro dû à Phytophthora colocasiae est l'affection la plus importante de cette culture au Cameroun depuis près d'une décennie. Cette étude a été réalisée pour évaluer l'activité antifongique des extraits des graines du laurier jaune scéniquement appelé Thevetia peruviana (Appocynacées) sur le développement in vitro de $P$. colocasiae et in vivo sur les fragments des feuilles infectées artificiellement. Méthodologie et résultats : les extraits de T. peruviana ont été préparés à partir des solvants : eau, méthanol, acétone et acétate d'éthyle aux concentrations respectives de 12,$5 ; 25 ; 50$ et $100 \mathrm{mg} / \mathrm{ml}$. Une solution de $20 \mu \mathrm{l}$ des fructifications de $P$. colocasiae isolé des feuilles infectées du cultivar de taro "Macumba» puis cultivé sur milieu V8-Agar, a été utilisée à une concentration de $5 \times 10^{4} \mathrm{sporanges} / \mathrm{ml}$. Pour les tests in vitro, des explants de $P$. colocasiae d'environ $0,8 \mathrm{~cm}$ de diamètre ont été déposés dans des boîtes de Pétri stériles contenant le milieu PDA supplémenté avec les différentes concentrations d'extraits, et mises en incubation à $23 \pm 1^{\circ} \mathrm{C}$ pendant sept jours pour l'évaluation de la croissance radiale. La sensibilité in vivo du pathogène aux extraits s'est faite sur des fragments de feuilles de taro par application de $20 \mu \mathrm{l}$ de suspension sporangiale de $P$. colocasiae suivie de $20 \mu \mathrm{l}$ d'extrait. Les résultats obtenus in vitro ont montré que les extraits aqueux, méthanoliques et à l'acétate d'éthyle ont totalement inhibé la croissance de l'agent pathogène à 25 $\mathrm{mg} / \mathrm{ml}$, tandis que l'extrait à l'acétone a inhibé la croissance à $12,5 \mathrm{mg} / \mathrm{ml}$. Les fragments de feuilles inoculés au champignon et traités à l'extrait à l'acétone n'ont pas développé les symptômes du mildiou après cinq jours d'inoculation du pathogène.

Conclusion et application potentielle : L'extrait des graines de T. peruviana à l'acétone à la dose de 12,5 $\mathrm{mg} / \mathrm{ml}$ a inhibé totalement la croissance radiale de $P$. colocasiae in vitro. Cet extrait s'est révélé efficace contre $P$. colocasiae et peut donc constituer une alternative de lutte contre le mildiou du taro. L'activité de cet extrait était comparable à celle du fongicide synthétique de référence (le Callomil plus 72 WP) utilisé. Les extraits bruts des graines de T. peruviana renfermeraient un grand nombre de composés bioactifs, qui une 
fois purifiés, pourraient présenter une activité antifongique assez levée au même titre que les fongicides synthétiques. Cette étude préliminaire constitue une base pour des essais futurs dans les conditions naturelles.

Mots clés : extraits bruts, Thevetia peruviana, activités antifongiques, Phytophthora colocasiae, Colocasia esculenta.

\begin{abstract}
Evaluation of antifungal activities of Thevetia peruviana extracts against Phytophthora colocasiae, causal agent of late blight of taro (Colocasia esculenta (L.) Schott) in Cameroon.

Objective: Taro leaf blight caused by Phytophthora colocasiae is the most devastating disease in taro production in Cameroon for one decade now. This study was conducted to evaluate the antifungal activities of extracts from Thevetia peruviana (yellow oleander) seeds on the in vitro growth of the fungus as well as on detached taro leaf fragments infected artificially.

Methodology and Results: Aqueous, methanolic, acetone and ethlyl acetate extracts of $T$. peruviana were prepared and used at concentrations of $12.5,25,50$ and $100 \mathrm{mg} / \mathrm{ml}$. P. colocasiae was isolated from an infected taro leaf cultivar "Macumba or lbo coco" in V8 agar medium and maintained in pure culture from which a suspension of $5 \times 10^{4}$ sporangia/ml was prepared. Mycelial fragments of $P$. colocasiae of about 0.8 $\mathrm{cm}$ in diameter were cut and placed in sterile Petri dishes containing Potato Dextrose Agar (PDA) medium supplemented with different concentrations of plant extracts and incubated at $23 \pm 1^{\circ} \mathrm{C}$ for seven days for the evaluation of the radial growth. In vivo sensitivity of the pathogen to plant extracts was done by application of $20 \mu \mathrm{l}$ of sporangial suspension on taro leaves followed by $20 \mu \mathrm{l}$ of each extract. The results obtained showed that the methanolic, ethyl acetate, and aqueous extracts completely inhibited the growth of the pathogen at $25 \mathrm{mg} / \mathrm{ml}$ while total inhibition of the pathogen was obtained with acetone extract at $12.5 \mathrm{mg} / \mathrm{ml}$. No symptoms were observed on leaf fragments that received a drop of the fungus and acetone extract.

Conclusion and potential application: The acetone extract at the concentration of $12.5 \mathrm{mg} / \mathrm{ml}$ totally inhibited the in vitro radial growth of $P$. colocasiae and significantly delayed the development of the disease on leaf fragments. This extract, active against $P$. colocasiae could be used as alternative to fungicides for the control of taro leaf blight. This activity was comparable to that of the reference fungicide used: Callomil plus 72 WP. These extracts are still crude and may contain a large number of different compounds, which after purification could present even a better activity than the fungicides used. This preliminary study provides a base line for future trials in natural conditions in greenhouse and in the field.
\end{abstract}

Keywords: Crude extracts, Thevetia peruviana, antifungal activities, Phytophthora colocasiae, taro.

\section{INTRODUCTION}

Le taro (Colocasia esculenta (L.) Schott) (Figure 1a) est un important aliment de base ou de subsistance pour de millions d'habitants dans les pays d'Afrique, d'Asie et d'Amérique Centrale (Mishra et al., 2008 ; Tsopmbeng et al., 2014b). La production mondiale est estimée à environ 10,64 millions de tonnes sur une surface cultivée de 1,67 millions d'hectares (FAOSTAT, 2020). Par ailleurs, $77 \%$ de la production mondiale de taro proviennent de l'Afrique subsaharienne (FAOSTAT, 2020). La feuille de taro est le quatorzième légume le plus consommé dans le monde (Rao et al., 2010). Le Cameroun est le troisième producteur mondial de taro, et le deuxième plus grand producteur en Afrique après le Nigéria avec une production de 1,9 millions de tonnes sur 7,87 millions de tonnes pour toute l'Afrique soit $24,14 \%$ de la production africaine (FAOSTAT, 2020). Le taro est cultivé dans toutes les régions du Cameroun pour ses feuilles et tubercules qui possèdent de bonnes qualités nutritives : amidon (73 à $80 \%$ ) très digeste avec une teneur en amylose variant entre 19 et $24 \%$ (Jane et al., 1992) et 30,62 \% (Elvina et al., 2005). II est ainsi plus riche en amylose que le manioc (16, $89 \%$ ) ou le maïs (22,4\%) (Liu et al., 1997); vitamine C (qui varie entre $37-140 \mathrm{mg} / 100 \mathrm{~g}$ de 
feuille fraiche); substances pro vitaminiques $A$ (carotènes et caroténoïdes) varient de 0,5 à 7,3 $\mathrm{mg} / 100 \mathrm{~g}$ par rapport à la matière fraîche (CPS, 1993). II occupe une place importante lors des cérémonies traditionnelles chez certaines populations africaines et asiatiques (Lyonga et Nzietchueng, 1991 ; Onwueme, 1999; Caillon, 2005). Les tubercules et les feuilles de taro ont aussi des vertus médicinales contre la tuberculose, les ulcères, les congestions pulmonaires et les infections fongiques (Misra et Sriram, 2002 ; Binoy et al., 2010). L'amidon très digeste des tubercules fait du taro un excellent aliment pour les diabétiques (Imar, 2011). Malgré son importance économique, alimentaire et socioculturelle, la culture du taro au Cameroun souffre de plusieurs contraintes

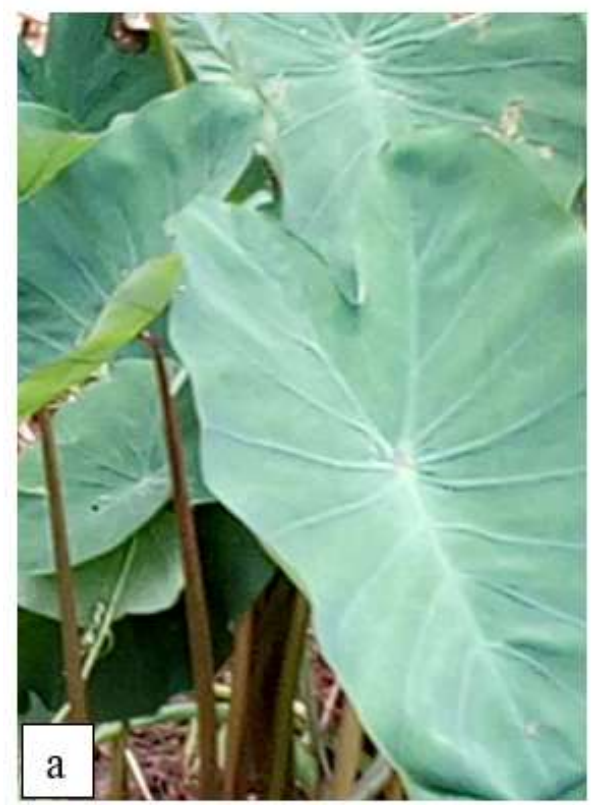

biotiques principalement les maladies épidémiques. L'une des plus importantes est le mildiou causé par Phytophthora colocasiae (Guarion, 2010). La maladie affecte principalement les feuilles du taro (Figure $1 \mathrm{~b}$ ), et peut détruire complètement les cultivars sensibles en moins de 10 jours et occasionner des pertes de rendements de l'ordre de 50 \% (Gadre et Joshi, 2003 ; Fullerton et Tyson, 2004 ; Brooks, 2005). Cette perte de rendement affecte de manière remarquable les revenus des paysans de même que la sécurité alimentaire des populations humaines. Le pH 7 et la température de $27^{\circ} \mathrm{C}$ sont les conditions optimales de croissance de l'agent pathogène en champ (Tsompbeng et al., 2014a).

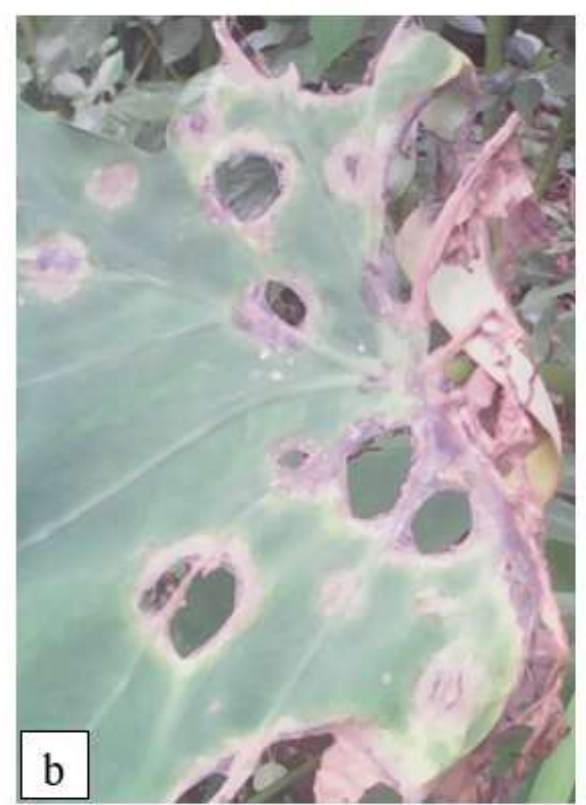

Figure 1 : Appareil végétatif et symptômes du mildiou du taro. (a) : plant sain de Colocasia esculenta, (b) : symptômes du mildiou du taro à la surface supérieure d'une feuille infectée.

Les stratégies de lutte contre $P$. colocasiae sont le plus souvent axés sur l'utilisation des fongicides chimiques à base de métalaxyl (Ashok et al., 1996 ; Carmichael et al., 2008); mais en raison des problèmes de résidus dans l'eau souterraine (Ndongo, 1999), du développement des résistances dans l'organisme cible, du coût élevé, et du danger pour l'homme et pour l'environnement (Ambang, 1996). Les méthodes de lutte alternatives sont de plus en plus envisagées (Ambang et al., 2007,
2009, 2011; Asseng et al., 2016, 2017). Actuellement, des efforts considérables sont orientés vers l'exploration des extraits de plantes à potentiel pesticide comme sources alternatives ou complémentaires aux pesticides synthétiques. Les extraits de plantes ont l'avantage d'être non seulement disponibles pour les paysans, mais aussi non toxiques et facilement biodégradables et donc sains pour l'environnement (Okigbo et Nmeka, 2005 ; Okigbo et Omdamiro, 2006). Certaines recherches 
ont montré l'efficacité des extraits de plantes sur Phytophthora infestans, agent causal du mildiou de la pomme de terre, de la tomate et de la morelle noire (Fontem et al., 2005 ; Goufo et al., 2010 ; Djeugap et al., 2011) et sur $P$. colocassiae (Tsopmbeng et al.,2014) mais aucune recherche n'a été faite sur l'effet des extraits de graines de Thevetia peruviana (laurier jaune) sur $P$. colocasiae au Cameroun. Les graines, feuilles, fruits et racines du laurier jaune (Thevetia peruviana) sont considérés comme des sources potentielles des

\section{MATÉRIEL ET MÉTHODES}

Matériel végétal et chimique : le matériel végétal était constitué des noyaux de graines de T. peruviana collecté dans la ville de Yaoundé où l'arbre sert de clôture de maison (plante ornementale) et des feuilles de plants de C. esculenta collectées dans une plantation paysanne située dans la localité de Bafang dans le Département du Haut-Nkam à l'Ouest Cameroun et transportées au laboratoire de Phytopathologie de l'Université de Yaoundé I. Le matériel chimique était un produit de dénomination commerciale Callomil plus 72 WP dont la matière active est constituée de $12 \%$ de métalaxyl et 60 $\%$ d'oxyde de cuivre.

Préparation des extraits des graines de $T$. peruviana : la plante de Thevetia peruviana a été identifiée suivant la clé de systématique botanique de l'espèce en se référant à la version récente du code international de nomenclature botanique (Greuter et al., 2003) (Spichiger et al., 2002). Les fruits matures de T. peruviana ont été ramassés, les noyaux extraits des fruits ont été concassés et les amandes obtenues ont été séchées à température ambiante pendant 3 à 4 semaines au laboratoire puis écrasées à l'aide d'un moulin manuel pour obtenir une poudre. Les extraits organiques, ont été préparés par macération de $1 \mathrm{~kg}$ de poudre dans $5 \mathrm{~L}$ de solvant (méthanol, acétone, acétate d'éthyle) pendant $48 \mathrm{~h}$ puis filtrée. Les filtrats obtenus ont été concentrés à $60^{\circ} \mathrm{C}$ à l'aide d'un évaporateur rotatif et les extraits aux solvants obtenus ont été conservés au réfrigérateur à 4 ${ }^{\circ} \mathrm{C}$ jusqu'à leur utilisation. Pour l'extraction aqueuse, la poudre obtenue est emballée dans du tissu de mousseline et trempée directement dans l'eau pendant $12 \mathrm{~h}$ puis essorée pour extraire le maximum de produit (Stoll, 1994 ; Ambang et al., 2009, 2010). L'extrait aqueux ainsi préparé est directement utilisé. Les doses d'extraits de 12,$5 ; 25 ; 50 ; 100 \mathrm{mg} / \mathrm{ml}$ ont été obtenues composés biologiques actifs comme insecticides (Ambang et al., 2005; Ngoh Dooh, 2014b), fongicides (Ambang et al., 2010, 2011 ; Mboussi et al., 2016), virucides (Tewtrakul et al., 2002) et bactericides (Saxena and jain, 1990). C'est ainsi que le présent travail se propose d'évaluer l'efficacité des extraits aqueux et organiques de Thevetia peruviana sur la croissance de $P$. colocasiae in vitro et de tester la sensibilité de cet agent pathogène aux différents extraits sur les fragments de feuilles de taro (in vivo).

suivant une progression géométrie de raison 2 (DerradjiHeffaf, 2013) à partir d'une solution mère de $500 \mathrm{mg} / \mathrm{ml}$ partant sur une base de $36 \mathrm{~g}$ de poudre dans $72 \mathrm{ml}$ de solvant.

Le rendement d'extraction de chaque extrait a été calculé en utilisant la formule citée par Ngoh Dooh et al. (2014a) :

$$
R d \%=\frac{\text { Masse de l'extrait }}{\text { Masse de la poudre }} X 100
$$

Isolement et purification de Phythopthora colocasiae : Les feuilles infectées de la variété de taro «Macumba» portant les symptômes du mildiou récoltées dans les champs paysans n'ayant obtenues aucun traitement phytosanitaire, ont été découpées en fragments d'environ $2 \mathrm{~cm}^{2}$ au niveau du front de croissance de l'agent pathogène et désinfectées superficiellement dans une solution d'hypochlorite de sodium à $5 \%$ pendant 2 minutes. Après trois rinçages à l'eau distillée stérilisée (EDS), les fragments ont été séchés sur du papier hydrophile puis déposés à raison de quatre fragments dans une boîte de Pétri contenant le milieu de culture $V 8$ gélifié supplémenté d'une solution d'antibiotiques composés de pénicilline $(250 \mathrm{mg} / \mathrm{l})$, d'ampicilline $(250 \mathrm{mg} / \mathrm{l})$ et de nystatine $(20 \mathrm{mg} / \mathrm{l})$ (Djeugap et al., 2009, Tsopmbeng et al., 2012). Après trois jours d'incubation au laboratoire à une température de $23 \pm 1^{\circ} \mathrm{C}$, des colonies du pathogène, visibles autour des fragments ont été prélevées et repiquées dans de nouvelles boîtes de Pétri contenant le milieu de culture PDA. Ce processus a été répété plusieurs fois jusqu'à l'obtention de cultures pures morphologiques du mycélium (non septé) et des fructifications (sporanges) telles que décrites par Brooks (2005) et Scot et al. (2011). 


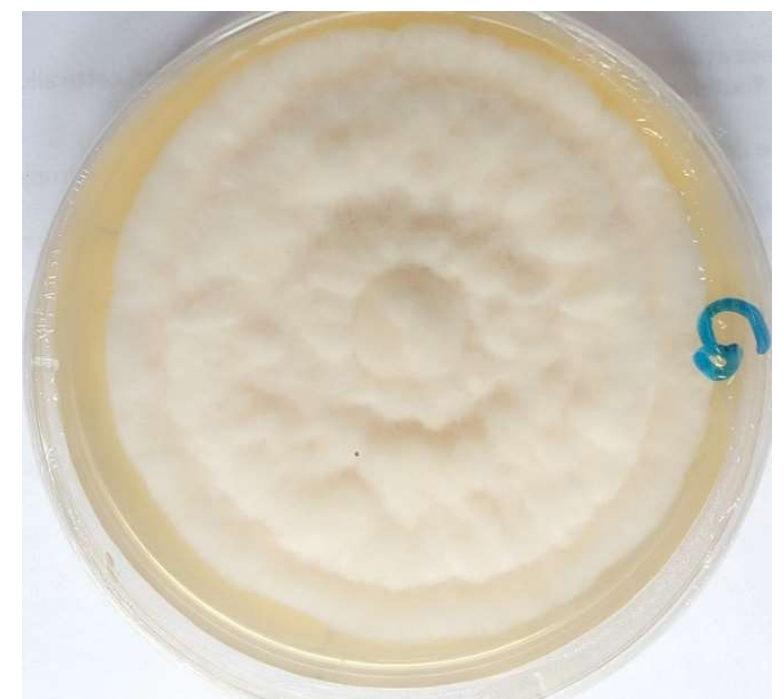

Figure 2 : Souche pure de Phytophtora colocassiae

Les isolats obtenus sont caractérisés suivant les critères morphologiques tels que la pathogénicité, la sporulation et la vitesse de croissance (Ondo, 2006).

Préparation de l'inoculum de $P$. colocasiae : Des cultures pures et fructifères de $P$. colocasiae âgées de 21 jours ont été prudemment brossées à l'aide d'un pinceau fin dans $20 \mathrm{ml}$ d'eau distillée stérile. La suspension sporangiale obtenue a été filtrée à la mousseline pour éliminer les fragments mycéliens. Une goutte de Tween 20 y a été ajoutée pour homogénéiser la suspension des spores qui par la suite a été quantifiée à $5 \times 10^{4}$ sporanges $/ \mathrm{ml}$ à l'aide d'un hemacytomètre puis conservée à $4{ }^{\circ} \mathrm{C}$ pendant 30 min dans un réfrigérateur pour stimuler la libération des zoospores (Xu et Kho, 1998, Zhu et al., 2001).

Évaluation in vitro de l'activité antifongique des extraits bruts: L'évaluation in vitro de l'activité antifongique des extraits s'est faite aux concentrations de 12,$5 ; 25 ; 50$ et $100 \mu \mathrm{l} / \mathrm{ml}$ pour les extraits organiques et aqueux à partir des solutions mères de $500 \mathrm{mg} / \mathrm{ml}$ pour chacun. Un fongicide de synthèse (Callomil plus 72
WP) a été utilisé comme témoin positif en prélevant dans un sachet de $50 \mathrm{~g}, 1 \mathrm{~g}$ de poudre pour $5 \mathrm{ml}$ d'eau distillée. Des explants mycéliens de $P$. colocasiae d'environ $8 \mathrm{~mm}$ de diamètre ont été prélevés à l'emportepièce sur une culture pure et fructifère âgée de sept jours et placés au centre de la boîte de Pétri contenant les milieux enrichis aux différents extraits ou au fongicide. Un témoin négatif non supplémenté en extrait a été élaboré. Chaque traitement a été répété 3 fois. L'incubation a été réalisée à $23 \pm 1^{\circ} \mathrm{C}$ sous une photopériode de 12/12 pendant une semaine. Une mesure journalière du diamètre de la croissance radiale de chaque explant mis en culture a été prise et s'est poursuivie jusqu'à ce que le mycélium remplisse les boîtes témoins. La croissance radiale de l'agent pathogène a été évaluée en mesurant deux diamètres perpendiculaires tracés au verso de la boîte de Pétri. La moyenne des deux mesures perpendiculaires ôtée du diamètre de l'explant représente la mesure de la croissance radiale du champignon. Elle est obtenue par la formule décrite par Dohou et al. (2004) (Figure 3):

$$
\mathrm{D}=\frac{\mathrm{D} 1+\mathrm{D} 2}{2}-\mathrm{D} 0
$$

Où : D0 est le diamètre de l'explant ; D1 et D2 sont les diamètres de culture mesurés dans les deux directions perpendiculaires. 
Boîte de Pétri de diamètre

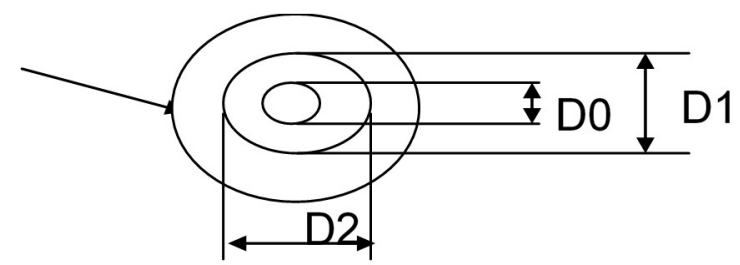

Figure 3 : Schéma de mesure de la croissance mycélienne en boîte de Pétri sur milieu V8.

Le pourcentage d'inhibition (I \%) dû à chaque extrait est évalué par rapport à la croissance mycélienne dans les boîtes témoins selon la formule développée par Dohou et al. (2004) :

$$
I \%=\frac{\text { Dto } \mathrm{mm}-\text { Dxi } \mathrm{mm}}{\text { Dto } \mathrm{mm}} X 100
$$

Avec I (\%) le pourcentage d'inhibition; Dto le diamètre moyen du lot témoin et Dxi le diamètre moyen des lots en présence de l'extrait.

Évaluation de l'activité antifongique des extraits sur les fragments de feuilles de taro: Sur la base des résultats du test in vitro des différents extraits, la concentration de $25 \mathrm{mg} / \mathrm{ml}$ pour les extraits organiques et aqueux de $T$. peruviana a été sélectionnée pour les tests sur les fragments de feuilles. Les feuilles saines récoltées sur des plants âgés de 3 mois ont été lavées, désinfectées dans une solution d'eau de Javel à $5 \%$ pendant $2 \mathrm{~min}$, puis rincées deux fois à l'eau distillée stérilisée. Elles ont été découpées en fragments d'environ $8 \mathrm{~cm}$ de diamètre et déposées dans des boîtes de Pétri en plastique de $9 \mathrm{~cm}$ de diamètre. Un volume de $20 \mu \mathrm{l}$ d'extrait de concentration $25 \mathrm{mg} / \mathrm{ml}$ a été déposé au préalable sur la face inférieure des fragments foliaires suivie de $20 \mu \mathrm{l}$ de suspension sporangiale de $P$. colocasiae quantifiée à $5 \times 10^{4}$ sporanges $/ \mathrm{ml}$. Du coton

\section{RÉSULTATS}

Rendement des extractions : le rendement, le volume, la couleur et l'aspect des différents extraits obtenus dépendent du solvant d'extraction utilisé (Tableau I). L'extraction au méthanol a donné le rendement le plus élevé : $35 \%$ et celle à l'eau le rendement le plus faible : $20,33 \%$. Avec les deux autres solvants: l'acétate d'éthyle et l'acétone, on a obtenu des rendements imbibé d'eau distillée stérilisée a été placée à la base de ces fragments de feuilles pour maintenir l'humidité dans la boîte pendant l'expérience. Les fragments témoins ont reçu $20 \mu \mathrm{l}$ d'inoculum, $20 \mu \mathrm{l}$ d'eau distillée stérile (témoin négatif), $20 \mu \mathrm{l}$ d'inoculum et $20 \mu \mathrm{l}$ de bouillie fongicide de concentration $0,025 \mathrm{mg} / \mathrm{ml}$ (témoin positif). Après 5 jours d'incubation à $23 \pm 1^{\circ} \mathrm{C}$, la surface des lésions développées sur les fragments a été mesurée en comptant le nombre de carrés couverts par la lésion formée à l'aide du papier millimétré. L'expérience a été rangée dans un dispositif factoriel $4 \times 1$ (4 extraits $\mathrm{x}$ 1dose) avec trois répétitions.

Analyse statistique : Les pourcentages d'inhibition de la croissance radiale du pathogène ont été transformés en probits et les valeurs obtenues ont été régressées sur le logarithme de la concentration des extraits végétaux. L'efficacité des extraits a été évaluée sur la base de la valeur de concentration équivalente de $50 \%\left(\mathrm{CE}_{50}\right)$ et $90 \%\left(\mathrm{CE}_{90}\right)$ déterminées après 8 jours de croissance selon la formule élaborée par Finney (1971). Les données de pourcentages d'inhibition, des $\mathrm{CE}_{50}, \mathrm{CE}_{90}$ et de la surface des lésions ont été soumises à une analyse de variance en utilisant le logiciel d'analyse $R$ version 5.1 .0 et les moyennes ont été séparées par le test multiple de Duncan au seuil de probabilité $5 \%$.

intermédiaires, soit 28,5 et $23,3 \%$ respectivement. Les extraits à l'acétate d'éthyle sont huileux et de couleur jaunâtre tandis que les extraits au méthanol et à l'acétone ont un aspect visqueux et de couleur marron. L'extrait aqueux a un aspect liquide et blanchâtre (tableau 1).

Tableau 1 : Rendement des extractions (\%) et caractéristiques des extraits pour $1 \mathrm{~kg}$ de graines.

\begin{tabular}{lll}
\hline Extrait à & Rendement & Caractéristiques \\
\hline Acétate d'éthyle (EAE) & 28,5 & Huileux et de couleur jaune pâle \\
Acétone (EAc) & 23,3 & Marron et visqueux \\
Méthanol (EM) & 35 & Marron clair et très visqueux \\
Eau (Eaq) & 20,33 & Liquide et blanchâtre \\
\hline
\end{tabular}


Effet des extraits de $T$. peruviana sur la croissance in vitro de $P$. colocasiae : les extraits de graines testés ont inhibé de manière significative la croissance radiale de $P$. colocasiae. Le développement des colonies fongiques dans les boîtes de Pétri ayant reçu les concentrations élevées des extraits était fortement inhibé et quasiment nul selon les différents extraits

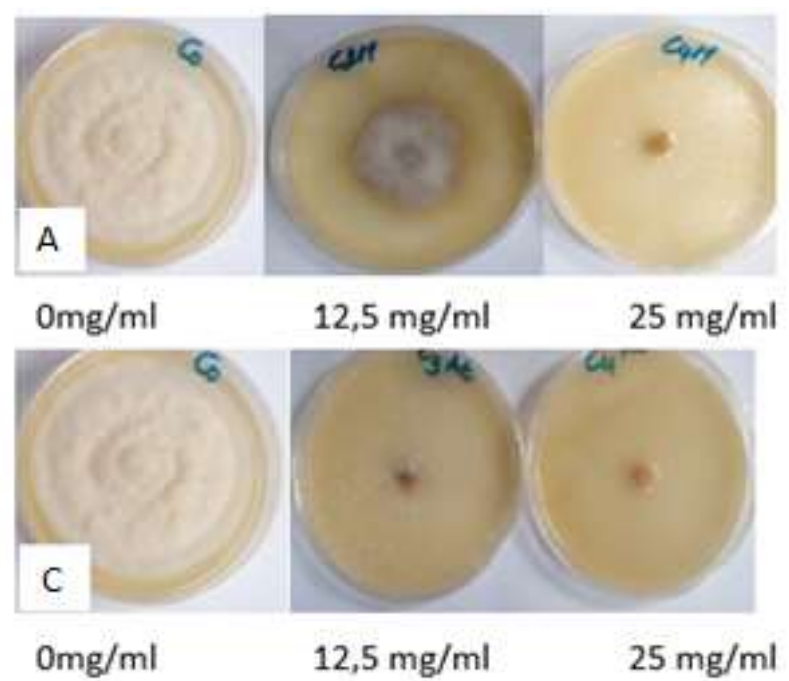

testés (figure 4). L'extrait à l'acétone a totalement inhibé la croissance du pathogène à $12,5 \mathrm{mg} / \mathrm{ml}$ (Figure $4 \mathrm{C}$ ) tandis que l'inhibition totale a été obtenue à la concentration de $25 \mathrm{mg} / \mathrm{ml}$ pour l'extrait aqueux (Figure $4 B$ ), à l'acétate d'éthyle (Figure 4D) et au méthanol (Figure 4A). Par contre, dans les témoins, $P$. colocasiae remplissait le fond des boîtes de Pétri en 8 jours.
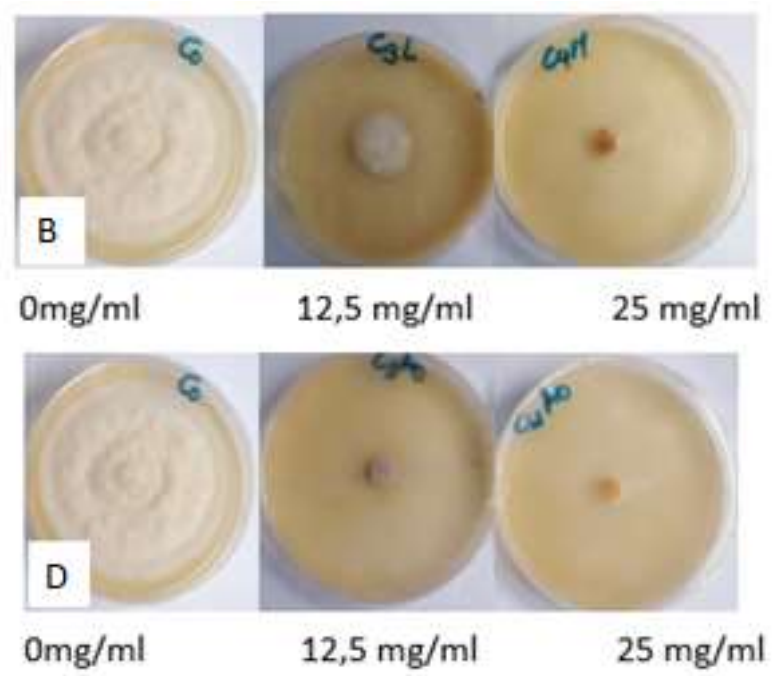

Figure 4 : Activité inhibitrice in vitro des extraits de Thevetia peruviana sur la croissance radiale de $P$. colocasiae après 8 jours d'incubation sur milieu PDA ; A : Extrait au méthanol, B : Extrait aqueux, C : Extrait à l'acétate d'éthyle et D : Extrait à l'acétone.

Pourcentage d'inhibition des extraits : le pourcentage d'inhibition de la croissance mycélienne de $P$. colocasiae a varié avec l'augmentation de la concentration des extraits aqueux et organiques. Les extraits aqueux, à l'acétate d'éthyle et au méthanol ont présenté un une inhibition de $100 \%$ à la concentration de $25 \mathrm{mg} / \mathrm{ml}$; par contre, avec l'extrait à l'acétone l'inhibition totale (100\%) a été observée avec la concentration de $12,5 \mathrm{mg} / \mathrm{ml}$ (Tableau 2). Les boîtes de Pétri non traitées ont des pourcentates d'inhibition nuls. L'inhibition totale de la croissance du pathogène observée à la concentration de $12,5 \mathrm{mg} / \mathrm{ml}$ par l'extrait à l'acétone a été semblable à celle obtenue avec le Callomil Plus (Tableau 2).

Tableau 2 : Pourcentage d'inhibition (\%) des extraits aqueux et organiques et du fongicide de synthèse (Callomil Plus) sur la croissance mycélienne de P. colocasiae.

\begin{tabular}{|c|c|c|c|c|c|}
\hline \multirow{2}{*}{ Concentration (mg/ml) } & \multicolumn{5}{|c|}{ Pourcentage d'inhibition (\%) selon les solvants } \\
\hline & Eau & Méthanol & Acétate & Acétone & Fonqicide \\
\hline 12,5 & $74,04^{c}$ & $61,37^{d}$ & $88,16^{b}$ & $100^{\mathrm{a}}$ & $100^{\mathrm{a}}$ \\
\hline 25 & $100^{a}$ & $100^{a}$ & $100^{\mathrm{a}}$ & $100^{a}$ & $100^{a}$ \\
\hline 50 & $100^{a}$ & $100^{a}$ & $100^{a}$ & $100^{a}$ & $100^{a}$ \\
\hline
\end{tabular}

*Les moyennes dans la même colonne suivies de lettres identiques ne présentent pas de différences significatives selon le Test de Duncan au seuil de probabilité de $5 \%$. 
Concentrations équivalentes $\mathrm{CE}_{50}$ et $\mathrm{CE}_{90}$ des extraits : Les concentrations équivalentes $\mathrm{CE}_{50}$ et $C E_{90}$ ont été plus élevées avec l'extrait au méthanol $(18,37)$, suivi de l'extrait aqueux $(15,20)$ et l'extrait à l'acétate d'éthyle (15). Les valeurs les plus faibles ont été obtenues avec l'extrait à l'acétone et le Callomil plus $(11,25)$ (Tableau 3).

Tableau 3. Les valeurs de $\mathrm{CE}_{50}$ et $C E_{90}(\mu \mathrm{g} / \mathrm{ml})$ sur la croissance mycélienne de $P$. colocasiae en fonction des extraits végétaux et du fongicide de synthèse.

\begin{tabular}{l|l|l}
\hline Extraits/fongicides de synthèse & $\mathbf{C E}_{50}$ & $\mathbf{C E}_{90}$ \\
\hline Acétate d'éthyle & $*$ & 15 \\
Acétone & $*$ & 11,25 \\
Méthanol & $*$ & 18,37 \\
Eau & $*$ & 15,20 \\
Fongicide & $*$ & 11,25 \\
\hline
\end{tabular}

${ }^{*}$ Représente les valeurs qui ne sont pas définies pour être à zéro statistiquement.

Effet des extraits de graines sur le développement in vivo du mildiou : Les symptômes du mildiou se sont timidement développés quatre jours après incubation sur les fragments de feuilles de taro détachées inoculés au $P$. colocasiae et traités aux extraits de $T$. peruviana. Par contre, dans les traitements témoins absolus, les nécroses se sont développées sur les fragments de feuilles de taro deux jours après inoculation du champignon. Aucun symptôme n'a été observé sur les fragments de feuilles ayant reçu le fongicide ou l'extrait à l'acétone (Figure 5). Les extraits à l'acétone ont significativement inhibé le développement du mildiou sur les fragments de feuilles de taro au même titre que les fongicides de synthèse à base du Métalaxyl+Oxyde de Cuivre. Par contre, les fragments de feuilles traités aux extraits aqueux et méthanoliques ont présenté respectivement des lésions de 0,17 et $0,21 \mathrm{~cm}^{2}$, lesquelles ont été significativement inférieures $(P<0,05)$ à celles obtenues sur les fragments de feuilles sans extraits et fongicides $\left(4,29 \mathrm{~cm}^{2}\right)$ (Tableau 4$)$.

Tableau 4 : Surface des lésions $\left(\mathrm{cm}^{2}\right)$ formées sur les fragments de feuilles de taro, 5 jours après application de l'inoculum, des extraits végétaux et des fongicides de synthèse.

\begin{tabular}{lc}
\hline Extraits/fongicides de synthèse & Surface des lésions $\left(\mathbf{c m}^{2}\right)$ \\
\hline Acétate d'éthyle & $0,10^{\mathrm{c}}$ \\
Acétone & $0,0^{\mathrm{d}}$ \\
Méthanol & $0,21^{\mathrm{b}}$ \\
Aqueux & $0,17^{\mathrm{b}}$ \\
Fongicide & $0,0^{\mathrm{d}}$ \\
Témoin & $4,29^{\mathrm{a}}$ \\
\hline
\end{tabular}

*Les moyennes dans la colonne suivies de la même lettre ne sont pas significativement différentes selon le test de Duncan à $5 \%$. 

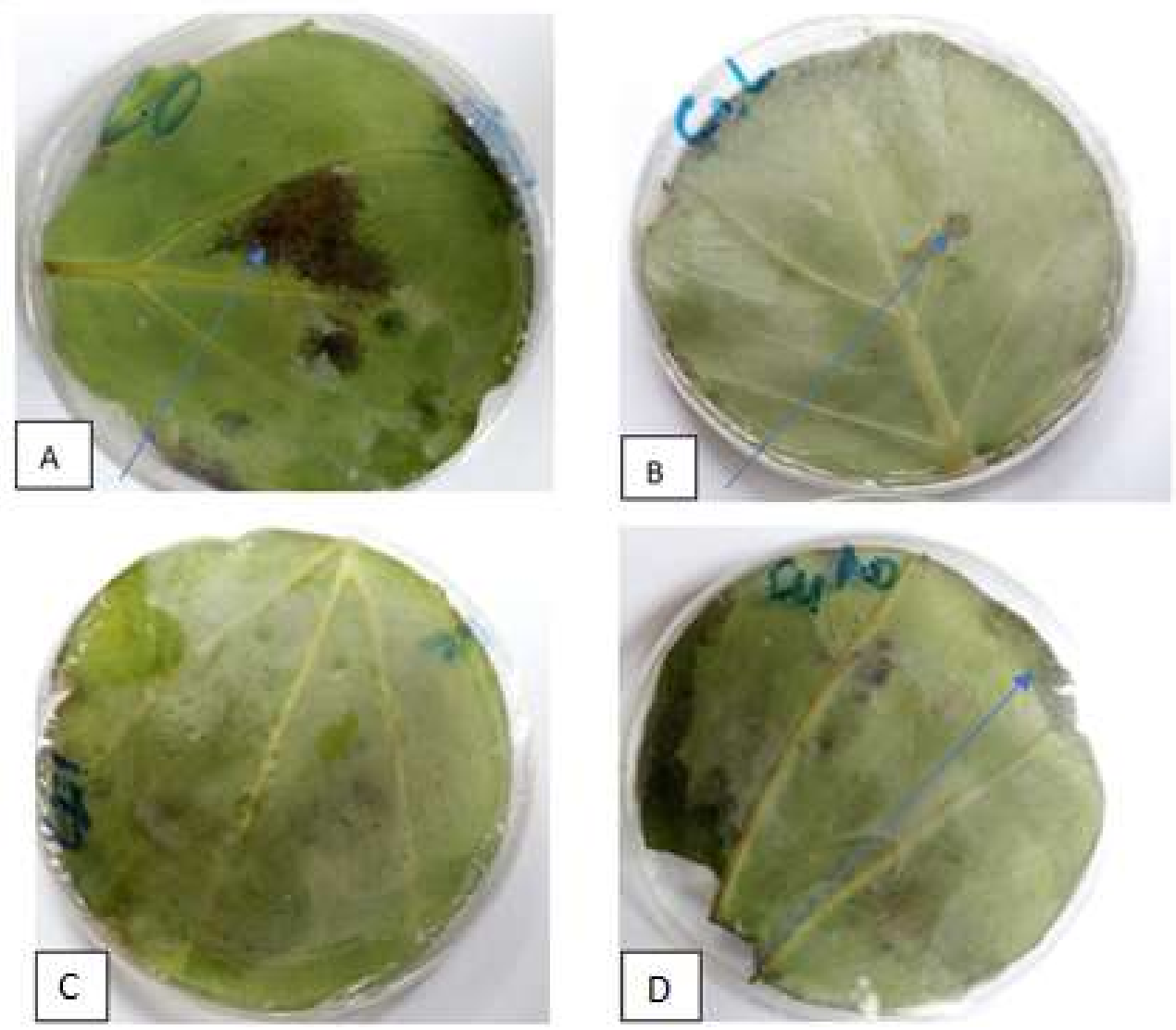

Figure 5 : Développement du mildiou du taro sur fragment de feuilles détachées sous l'influence des extraits aqueux et organiques de $T$. peruviana 5 jours après inoculation de $P$. colocasiae ;

$A$ : Témoin, $B$ : Extrait aqueux, $C$ : Extrait à l'acétone et $D$ : Extrait au méthanol.

\section{DISCUSSION}

Rendements des extractions : ils ont varié d'un solvant à un autre. La différence de rendement observée entre les extraits aqueux et organiques pourrait s'expliquer par la nature des solvants. Selon Ciulei (1981), les solvants organiques fixeraient plus de composés par rapport à l'eau et augmenteraient par conséquent le rendement d'extraction. En outre, la forte polarité des solvants tels que le méthanol permet qu'il soit plus efficace dans l'extraction de nombreux composés (Muhammad et al., 2013). Ngoh Dooh (2006) et Ambang et al. (2010) après extraction utilisant les mêmes quantités de poudre de $T$. peruviana avec les mêmes volumes de solvant au Cameroun, avaient obtenu un rendement de 7,44\% avec le méthanol et $9,43 \%$ avec l'acétate d'éthyle contre 35 et $28,5 \%$ respectivement dans la présente étude. Ces différences seraient dues par les conditions environnementales, la période de récolte et l'âge du matériel végétal comme l'ont rapporté dans des essais similaires par Svoboda et Hampson (1999) et Smallfield (2001).
Effet des extraits de graines de $T$. peruviana sur la croissance in vitro de $P$. colocasiae: Les différents extraits testés ont réduit de manière significative le développement des colonies de $P$. colocasiae par rapport au témoin. Cette réduction a été plus prononcée avec les extraits organiques (acétate d'éthyle et acétone). Ces extraits contiendraient des substances bioactives qui inhiberaient ou retarderaient la croissance du champignon. En effet, Pamo et al. (2003) et Ngoh Dooh (2014a) ont rapporté que les extraits végétaux d'un certain nombre de plantes contiennent des composés tels que les tanins, les flavonoïdes et les alcaloïdes qui sont dotés de propriétés fongicides. Les différentes concentrations d'extraits ont influencé de façon significative la croissance radiale du champignon. Les concentrations élevées (25 et $50 \mathrm{mg} / \mathrm{ml}$ ) étant plus efficaces dans l'inhibition du champignon. Des résultats semblables sur l'activité antifongique de certains de ces extraits avaient été rapportés par Tsompbeng et al. (2014b) en utilisant les extraits aqueux et méthanoliques 
de Laggera pterodonta et de Cupressus lusitanica, sur le même pathogène.

Pourcentage d'inhibition des extraits : Les pourcentages d'inhibition des extraits végétaux sur la croissance du pathogène ont également varié avec l'augmentation des concentrations et la nature des solvants d'extraction. A des fortes concentrations, tous les extraits ont présenté un arrêt total du développement de $P$. colocasiae in vitro comparable au résultat du fongicide de synthèse à base du métalaxyl + oxyde de cuivre. Des résultats semblables sur l'activité antifongique des extraits de L. pterodonta et de $C$. Iusitanica ont été rapportés par Djeugap et al. (2011) sur $P$. infestans, agent causal du mildiou de la morelle noire. Par ailleurs, à de faibles concentrations, les extraits organiques ont été plus actifs que les extraits aqueux. Le même constat a été fait par Tsompbeng et al. (2014b) dans des essais similaires. Ce décalage d'efficacité entre les extraits aqueux et organiques pourrait être attribuée à une différence de concentration des composés chimiques lors du processus d'extraction. Selon Bougandoura et Bendimerad (2012), les solvants organiques permettent une meilleure extraction des composés tels que les flavonoïdes et les terpénoïdes qui sont des molécules reconnues pour leur activité antifongique. En outre, l'eau agirait beaucoup plus sur des substances inactives qu'actives, alors que l'acétone plus sélectif agirait plus sur des substances actives contre le pathogène. Cette hypothèse corrobore avec les travaux d'Akhilesh et al. (2010) qui ont rapporté que l'extraction au méthanol était plus efficace sur l'activité antimicrobienne que celle à l'eau. En effet, ces extraits ont été rapportés par Ngoh Dooh et al. (2014a) de

\section{CONCLUSION}

La présente étude a montré que les extraits de $T$. peruviana ont inhibé la croissance radiale de $P$. colocasiae in vitro et retardé de façon significative le développement du mildiou sur les fragments de feuilles après cinq jours d'inoculation. Ces extraits se sont révélés actifs sur $P$. colocasiae et peuvent donc constituer une alternative dans la lutte contre le mildiou du taro. Bien que leur efficacité ait été comparable à

\section{REMERCIEMENTS}

Les auteurs remercient les responsables des laboratoires de Phytopathologie de l'Université de Yaoundé 1 et de l'Institut de Recherche Agricole pour le contenir de la thévetine qui est une substance antifongique. En termes des concentrations équivalentes de $\mathrm{CE}_{50}$ et $C E_{90}$, il ressort également que certains extraits organiques sont plus efficaces que les extraits aqueux suggérant ainsi qu'ils sont plus fongitoxiques.

Effet des extraits de graines sur le développement du mildiou sur les fragments de feuilles de taro : Les tests sur les fragments de feuilles de taro ont davantage confirmé l'efficacité des extraits organiques et aqueux. Aucune lésion n'a été observée 5 jours après inoculation sur des feuilles traitées à extrait à l'acétone. Cet extrait aurait des propriétés antifongiques similaires au fongicide de synthèse testé. Quant aux fragments de feuilles traités aux extraits aqueux, ils ont présenté les lésions quatre jours après inoculation contrairement au témoin où elles ont apparu au deuxième jour. Ceci suggère que l'extrait aqueux pourrait réduire la vitesse de développement de la maladie mais sans toutefois l'inhiber complètement. Des résultats similaires ont été obtenus par Tsompbeng et al. (2014b) en utilisant les extraits aqueux et méthanoliques de L. pterodonta et de C. Iusitanica, sur le même pathogène; Ambang et al. (2010), Ngoh Dooh et al. (2014b), Mboussi et al. (2016) utilisant les extraits des graines de $T$. peruviana sur $P$. megakaya et par Djeugap et al. (2011) sur $P$. infestans avec les extraits d'Eucalyptus saligna et de Callistemon viminalis respectivement sur la pomme de terre et la morelle noire en serre. Par contre, l'extrait au solvant organique tel que l'acétone a inhibé le développement de la maladie en supprimant totalement la germination des spores du pathogène d'où l'absence totale des symptômes plusieurs jours après inoculation.

celle du fongicide de référence (le Callomil plus 72 WP), ces extraits bruts pourraient contenir un grand nombre de composés qui, une fois purifiés, présenteraient une activité supérieure à celle des fongicides synthétiques. Les résultats obtenus dans ce travail constituent une base pour des essais futurs dans les conditions naturelles en serre et en champ.

Développement (IRAD) de Yaoundé qui ont bien voulu mettre à notre disposition les équipements nécessaires pour la réalisation de ce travail. 


\section{RÉFÉRENCES}

Akhilesh D., Neeraj M. \& Neha S. 2010. Antimicrobial Activity of Some Selected Vegetables. International Journal of Applied Biology and Pharmaceutical Technology, (3): 994-999.

Ambang Z.,1996. Efficacité de nouveaux pesticides contre le mildiou du doryphore de la pomme de terre, leur action sur les tissus de la plante. These de ph.D. université de Russie de l'Amitié des peuples.,Moscou. 128p.

Ambang Z., Ndongo B., Ngoh Dooh J.P. et Djilé B. 2005. Effet des extraits de graines de laurier jaune (Thevetia peruviana (Pers) K. schum sur les charançons (Sitophilus zemais Motsch) ravageurs des stocks. Bios prod.11(5783):1019-7702.

Ambang Z., Bekolo N., Petga E., Dooh J. P. N., Asanga A. 2007. Effect of crude extracts of Thevetia peruviana seeds on development of leaf spot disease of groundnut (Arachis hypogaea L.) caused by Cercospora sp. 8th African Crop Science Society Conference, El-Minia, Egypt, 22:797-800.

Ambang Z., Ndongo B., Amayana D., Djilé B., Ngoh J. P., Chewachong G.M. 2009. Combined effect of host plant resistance and insecticide application on the development of cowpea viral diseases. Austr. Jrn. Crp. Sc. 3(3):167-172.

Ambang Z., Ngoh Dooh., J. P., Essono G., Bekolo N., Chewachong1 G., Asseng C.C. 2010. Effect of Thevetia peruviana seeds extract on in vitro growth of four strains of Phytophthora megakarya. Plant Omics Journal. 3 (6) 204209.

Ambang Z., Essono G., Kosma P., Ndongo B. \& Ngoh J.P., 2011. Control of leaf spot disease cause by Cercospora sp on groundnut (Arachis hypogea) using methanolic extracts of yellow oleander (Thevetia peruviana) seeds. Austr. Jrn. Crp. Sci., 3(5.3): 227-232

Ashok B. \& Saikia U.N. 1996. Fungicidal management of leaf blight of Colocasia. International Journal of Tropical Agriculture, 14(4): 231-233.

Asseng C.C., Mvoe C.R., Ambang Z., and Monkam T.F. 2016. Influence of the Number of Watering and Fungicide Treatments on the Development of Phytophthora colocasiae (Racib) on Cocoyam (Xanthosoma sagittifolium) and Taro (Colocasia esculenta) Greenhouse in Cameroon. Int.J.Curr.Microbiol.App.Sci. 5 (8) : 100-112.
Asseng C.C., Ebongo L.E., Nanda D.G. L., Akono N.P., Mbida J.A., Ngono N.A., Ambang Z., Monkam T. F., Djouokep L.G. 2017. Study of Antagonistic Beneficial Microorganisms to Phytophtora colocasiae, Causal Agent of Taro Mildew (Colocasia esculenta (L.) Schott). Plant. $5(3): 51-60$.

Binoy B., Vinayaka H., Makeshkumar T. \& Jeeva M.L. 2010. Rapid Detection and Identification of Potyvirus Infecting Colocasia esculenta (L.) Schott by Reverse Transcription-Polymerase Chain Reaction. Journal of Root Crops 36 (1): 88-94.

Bougandoura N., Bendimerad N. 2012. Effet antifongiques des extraits aqueux et méthanolique de Satureja calamintha ssp (Nepeta) briq. Revue des Bio Ressources 2 : 1 7.

Brooks F.E. 2005. Taro leaf blight. The Plant Health Instructor. http://www.apsnet.org/edcenter/intropp/lesson s/fungi/Oomycetes/Pages/TaroLeafBlight.aspx Site visité le 15 novembre 2011.

Caillon S., 2005. Les taros du Vanuatu : que conserver et comment? Natures Sciences Sociétés 13 : 306-310.

Carmichael A., Harding R., Jackson G., Kumar S., Lal S.N., Masamdu R., Wright J. \& Clarke A.R., 2008. Taro Pest : an illustrated guide to pests and diseases of taro in the South Pacific. ACIAR Monograph No. 132, $76 \mathrm{p}$.

Ciulei I.1981. Methodology for Analysis of Vegetable Drugs. Pratical Manuals on the industrial utilization of medicinal and aromatic plants. Arta Grafica, Bucharest, Romania. 420 p.

CPS (Commission du Pacifique Sud), 1993. Le taro: un aliment pour tous. Fiche $\mathrm{N}^{\circ} 1$, Edition revue, 1993. Service de santé publique. Nouméa, Nouvelle-Calédonie.

Derradji-Heffaf D.F., 2013. Composition chimique et activité insecticide de trois extraits végétaux à l'égard de Sitophilus oryzae L. (Coleoptèra : Curculionidae). Thèse en sciences agronomiques. Elharrach. 92 p.

Djeugap J.F., Fontem D.A. \& Tapondjou A.L., 2009. Évaluation des milieux de culture pour la croissance de Phytophthora infestans, agent causal du mildiou chez la morelle noire. Biosciences Proceedings $15:$ :85-92. 
Djeugap J.F., Fontem D.A. \& Tapondjou A.L., 2011. Efficacité in vitro et in vivo des extraits de plantes contre le mildiou (Phytophthora infestans) de la morelle noire. Int. J. Biol. Chem. Sci. 5(6) : 2205-2213.

Dohou N., Yamani K., Badoc, A \& Douira, A., 2004. Activité antifongique d'extraits de Thymelea Iythroides sur trois champignons du riz. Bull. Soc. Pharm. Bordeaux. 143:31-38.

Elvina P., Forrest S., Emperatriz P.D. 2005. Caracterization of some properties of starches isolated from Xanthosoma sagittifolium (tannia) and Colocasia esculenta (taro). Carbohyd. Polym.; 60 (2); 139-145.

FAOSTAT., 2020. FAO Economic and Social Department. The Statistics Division. Major Food and Agricultural Commodities and Producers. http://faostat.fao.org/default.aspx, visited $4^{\text {th }}$ February 2020.

Finney D.F. 1971. Probit Analysis (3rd ed.). University Press: Cambridge.

Fontem D.A., Tamokou J.D.D., Teugwa M.C., Tapondjou A.L. \& Gumezoe M.Y.D. 2005. Évaluation de la bioefficacité des extraits végétaux sur la croissance in vitro de Phytophthora infestans, agent causal du mildiou des Solanacées. Pp. 123-125.In: Recueil des Communications Orales de la IXe Journée Scientifique du Réseau Biotechnologies Végétales, 4-7 octobre 2005. Lomé, Togo.

Fullerton R.A. \& Tyson J.L., 2004. The biology of Phytophthora colocasiae and implications for its management and control. Pp. 107-111. In: Secretariat of the Pacific Community (Ed.). Third Taro Symposium, 2003.

Gadre U.A. \& Joshi M.S. 2003. Influence of weather factors on the incidence of leaf blight of Colocasia.Plant Protection Science 11: 168170.

Goufo P., Fontem D.A. \& Ngnokam D. 2010. Evaluation of plant extracts for tomato late blight control in Cameroon. New Zealand Journal of Crop and Horticultural Science. $38:$ 171-176.

Greuter W., Mcneill J., Barrie F.R., Burdet H.M., Demoulin V, Filgueiras T.S., Nicolson D.H., Silva P.C., Skog J.E., Trehane P., Turland N.J. \& Hawksworth D.L. 2003. International Code of Botanical Nomenclature (St. Louis Code). Adopted by the XVth International Botanical
Congress St Louis. Koeltz Scientific Books: Königstein.

Guarion L. Taro leaf blight in Cameroon. Agricultural Biodiversity Weblog. 2010. Available:http://agro.Biodiver.se/2010/07/ taroleaf-blight-in-Cameroon/ (Accessed on 15 May 2012).

Imar D. S. 2011. Pratiques traditionelles, valeur alimentaire et toxicité du taro (Colocassia esculenta L. SCHOTT) produit au Tchad. Sciences agricoles. Université Blaise PascalClermont-Fernand II. Français. NNT : 2011CLF22153. tel-00719605.

Jane J., Shen L., Chen J., Lim S., Kasemsuwan T., Nip W.K. 1992. Physical and chemical studies of taro starches and flours. Cereal Chem. 69 (5) 528-535.

Liu H., Ransden L., Corke H. 1997. Physical properties and enzymatic digestibility of acetylated ae, wx and normal maize starch. Carbohydrate polymers, 34, 283-289.

Lyonga S. N. \& Nzietchueng S., 1991. Les taros et la crise alimentaire en Afrique. In : racines tropicales : Les plantes-racines et la crise alimentaire en Afrique. Compte rendu du troisième symposium triennal sur les plantes racines de la société internationale pour les plantes-racines tropicales-Direction Afrique, du 17 au 23 aout 1986.

Mboussi S.B., Ambang Z., Ndogho A., Ngoh Dooh J.P. and Manga E.F. 2016. In vitro Antifungal Potential of Aqueous Seeds Extracts of Azadirachta indica and Thevetia peruviana against Phytophthora megakarya in Cameroon. Jrn. Apl. L. Sc. Int. 4(4) : 1-12.

Misra R.S. \& Sriram S. 2002. Medicinal value and export potential of tropical tuber crops. Pp. 376-386. In: Govil J.N., Pandey J., Shivkumar B.G. \& Singh V.K. (Eds.). Series Recent Progress in Medicinal Plants, Crop Improvement, Production Technology and Commerce. USA.

Mishra A.K., Sharma K. \& Misra R.S. 2008. Effect of benzyl amino purine on the pathogen growth and disease development of taro leaf blight caused by Phytophthora colocasiae. Jrn. Plt. Pathol. 90(2): 191-196.

Muhammad Z., Sadia H., Komal R., Nasir R., Muhammad R., Zia-UI-Haq M. \& Vincenzo D. F. 2013. Antioxidant Potential and Oil Composition of Callistemon viminalis Leaves. Scientific World Journal. 10 :1155. 
Ndongo B. 1999. Lessivage et distribution spatiotemporelle du linuron et de l'imidaclopride dans les sols sableux sous culture de pomme de terre (Solanum tuberosum) à port-neuf (Quebec). Thèse Ph.D. Université de Laval. 176 p.

Ngoh Dooh, (2006). Effet des extraits bruts de Thevetia peruviana (Pers.) K. Schum. Sur quelques souches de Phytophthora megakarya Bras. et Grif. In vitro. Mémoire D.E.A. Université Yaoundé I. 51p.

Ngoh Dooh, J.P., Ambang, Z., Tih Ewola, A., Heu, A., Kosma, P., Maho yalen, E.J., Ghogomu Tih, R. 2014a. Screening and the effect of extracts of Thevetia peruviana on the development of colletotrichum gloeosporioides, causal agent of cassava anthracnose disease. E3 J. Agric. Res. Develop. 4(4): 54-65.

Ngoh Dooh, J.P., Ambang, Z., Ndongo B., Heu A., Kuate T. W. 2014b. Effect of extracts of Thevetia Peruviana (Pers.) k. Schum on development of phytophthora megakarya causal agent of black pod disease of cocoa. J. Appl. Biosci.77: 65646574.

Okigbo, R.N. \& Nmeka I.N. 2005. Control of Yam tuber rot with leaf Extracts of Xylopia aethiopica and Zingiber offinale. Afr. J. Biotech. 4(8): 804-807.

Okigbo R.N. \& Omodamiro O.D. 2006. Antimicrobial effect of leaf extract of pigeon pea (Cajanus cajan (L) Mill sp) on some human pathogen. $J$. Herbs, spices and Med. Plants 12 (1/2) : 117127.

Ondo A.S. 2006. Charactérisation de quelques isolats de $P$. megakarya agent causal de la pourriture brune des cabosses de cacaoyer (Theobroma cacao L.) Mémoire de DEA, Université de Yaoundé I. $58 \mathrm{p}$.

Onwueme I. 1999. Taro cultivation in Asia and the Pacific. Food and Agriculture. Organization of the United Nations Regional Office for Asia and the Pacific. Bangkok, Thailand. $15 \mathrm{p}$.

Pamo T.E., Tapondjou L., Temdonkeng F., Nzogang J.F., Djoukeng J., Ngandeu F. et Kana J. R. 2003. Effet des huiles essentielles des feuilles et des extrémités fleuries des Cupressus lussitanica sur la Tique (Rhipicephalus Lunulatus) à l'ouest Cameroun. Rev Acad des Sci du Cmr. 3(3) : 169-175.

Rao R., Hunter D., Eyzaguirre P., Mathews P. 2010. Ethnobotany and global diversity taro. In the global diversity of taro: Ethnobotany and conservation: Ramanatha Rao V, Mathews PJ, Ezyaguire PB, Hunter D, Eds. Bioversity International: Rome, Italy:2-5.

Saxena V.K., Jain S.K. 1990. Thevetia peruviana kernel oil: a potential Bactericidal agent. Fitoterapia 61: 348-349.

Scot N., Brooks F.E. \& Glenn T. 2011. Taro Leaf Blight in Hawai'i. University of Hawai'i at Mänoa, Plant Disease 71: 1-14.

Smallfield B. 2001. Introduction to growing herbs for essential oils, medicinal and culinary Purposes. Crops and Food Rese (45) : 1-4.

Spichiger R., Savolainene V., Figeat M. \& Jeanmonod D. 2002. Botanique systématique des plantes à fleurs. Presses polytechniques et universitaires romandes.

Stoll,1994. Protection naturelle des végétaux en zone tropicale. CTA. AGRECOL.95-99.

Svoboda K.P. \& Hampson J.B. 1999. Bioactivity of essential oils of selected temperate aromatic plants, antibacterial antioxidant, antiinflammatory and other related pharmacological activities Plant Biology Department, SAC Auchincruive, Ayr, Scotland. UK.

Tewtrakul S., Nakamura N., Hattori M., Fugiwara T., Suparita T. 2002. Flavonone and flavonol glycosides from leaves of Thevetia peruviana and their HIV-1 reverse transcriptase and HIV1 integrase Inhibitory activities. Chem. Pharm. Bull. 50 : 630-635.

Tsopmbeng G.R., Fontem D.A. \& Yamdé K.F. 2012. Evaluation of culture media for growth and sporulation of Phytophthora colocasiae Racib., causal agent of taro blight. Int. J. Biol. Chem. Sci. 6(4): 1566-1573.

Tsopmbeng G.R., Lienou J.A., Megaptche C.J.P.\& Fontem D.A. 2014a. Effet of $\mathrm{pH}$ and temperature levels on in vitro growth and sporulation of Phytophthora colocasiae, taro leaf blight pathogen. Int. Jrn. Agro. Agri. Rrch. 4(4): 202-206.

Tsopmbeng N. G., Megatche C.J. P., Lienou J. A., Yaouba A., Djeugap F. J. \& Fontem D. A., 2014b. Évaluation des activités antifongiques des extraits de plantes contre Phytophthora colocasiae, agent causal du mildiou du taro (C. esculenta (L) Schott). J. Appl. Biosci. 81 :72217232.

Xu X.L. \& Ko W.H. 1998. A quantitative confined inoculation method for studies of pathogenicity 
Essomé et al., J. Appl. Biosci. 2020 Évaluation des activités antifongiques des extraits de graines de Thevetia peruviana contre Phytophthora colocasiae (Oomycètes) agent causal du mildiou du taro (Colocasia esculenta (L.) Schott)

of fungi on plants. Bot. Bull. Acad. Sin. 39: 187190.

Zhu J., Zhang Z. \& Yang Z. 2001. General research methods on pathogen of potato late blight
(Phytophthora infestans). Jrn Agri. Sci. 24 : 112-114. 